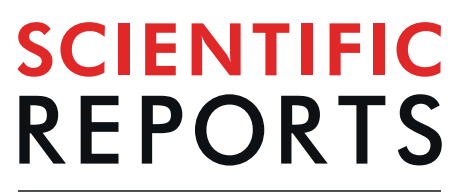

\title{
Clinical and economic impact of methicillin-resistant Staphylococcus aureus: a multicentre study in China
}

\author{
Xuemei Zhen ${ }^{1,2,3}$, Cecilia Stålsby Lundborg $\mathbb{1}^{3}$, Meiling Zhang ${ }^{4}$, Xueshan Sun ${ }^{1}$, Yuanyuan $\mathrm{Li}^{1}$, \\ Xiaoqian $\mathrm{Hu}^{1}$, Shuyan $\mathrm{Gu}^{1}$, Yuxuan $\mathrm{Gu}^{1}$, Jingming $\mathrm{Wei}^{1}$ \& Hengjin Dong $\mathbb{1}^{1,5^{*}}$
}

Methicillin-resistant Staphylococcus aureus (MRSA) has become a serious threat to global health. In China, the proportion of S. aureus isolates that were MRSA was 44.6\% in 2014. The clinical and economic impact of MRSA in China remains largely uninvestigated. This study aims to compare the differences in hospital costs, length of hospital stay, and hospital mortality rate between MRSA and methicillin-susceptible $S$. aureus (MSSA) colonization or infection and between MRSA cases and those without an S. aureus infection. A retrospective and multicentre study was conducted in four tertiary hospitals in China between 2013 and 2015. Inpatient characteristics and hospital costs were collected from electronic medical records. We conducted propensity score matching (PSM) to eliminate selection bias by balancing the potential confounding variables between the two groups. The main indicators included hospital costs, length of hospital stay, and hospital mortality rate. A total of 1,335 inpatients with MRSA, 1,397 with MSSA, and 33,606 without an S. aureus infection were included. PSM obtained 954 and 1,313 pairs between the MRSA and MSSA groups and between the MRSA and S. aureus-free groups, respectively. After PSM, MRSA colonization or infection is associated with an increased total hospital cost ranging from $\$ 3,220$ to $\$ 9,606$, an excess length of hospital stay of 6 days-14 days, and an attributable hospital mortality rate of $0-3.58 \%$. Between the MRSA and MSSA groups, MRSA colonization or infection was significantly associated with a higher total hospital cost and longer length of hospital stay among survivors but not among non-survivors; however, there were no differences in the hospital mortality rate between these two groups. Between the MRSA and the S. aureus-free groups, MRSA colonization or infection was significantly associated with an increased total hospital cost, a prolonged length of hospital stay and a higher hospital mortality rate among both survivors and non-survivors. It is critical to quantify the clinical and economic impact of MRSA to justify resource allocation for the development of strategies to improve clinical outcomes and to reduce the economic burden.

Staphylococcus aureus is an important gram-positive bacterium in both community- and health-care-associated infections and can be resistant or susceptible to the usual antibiotics used to treat it (oxacillin or cefoxitin), namely, methicillin-resistant $S$. aureus (MRSA) or methicillin-susceptible $S$. aureus (MSSA), respectively ${ }^{1,2}$. MRSA has become a serious threat to global health and is responsible for a range of infections, from skin and wound infections to pneumonia and bloodstream infections. It is commonly associated with significant morbidity, hospital mortality, length of stay and economic burden ${ }^{1}$. In all World Health Organization (WHO) regions, the overall proportion of $S$. aureus isolates that were MRSA exceeded $20 \%$ and even exceeded $80 \%$ in some reports ${ }^{3}$. In China, there was a marked decrease in the proportion of MRSA from $69 \%$ in 2005 to $44.6 \%$ in $2014^{4}$, even though it is still higher than other common multi-drug resistant bacteria ${ }^{5}$.

Many studies have explored the clinical and economic impact of MRSA ${ }^{6-24}$. However, some studies were conducted more than a decade ago, limiting their application to the current clinical environment $6,7,9,10,15,16,18-22$.

\footnotetext{
${ }^{1}$ Center for Health Policy Studies, School of Public Health, Zhejiang University School of Medicine, Hangzhou, China. ${ }^{2}$ School of Health Care Management, Shandong University, 44 Wenhuaxi Rd., Jinan, Shandong, P.R. China. ${ }^{3} \mathrm{Global}$ Health-Health Systems and Policy (HSP): Medicines, focusing antibiotics, Department of Public Health Sciences, Karolinska Institutet, Stockholm, Sweden. ${ }^{4}$ Department of Pharmacy, Tongde Hospital of Zhejiang Province, 234 Gucui Road, Hangzhou, 310012, China. ${ }^{5}$ The Fourth Affiliated Hospital Zhejiang University School of Medicine, No. N1, Shancheng Avenue, Yiwu City, Zhejiang, China. *email: donghj@zju.edu.cn
} 
Distinct findings are presented in published literature ${ }^{25,26}$. It was reported that compared with patients with MSSA colonization or infection, those with MRSA are significantly associated with higher hospital costs ${ }^{20,23,27,28}$, higher hospital mortality rates ${ }^{10,27,29}$, and longer length of hospital stays ${ }^{27,30}$; however, in some reports, there were no differences among clinical and economic outcomes between these two groups ${ }^{30,31}$. Moreover, the costs for MSSA infection were higher than those for MRSA cases in one study ${ }^{13}$. A few studies compared the differences in clinical and economic outcomes between MRSA cases and those without an S. aureus infection ${ }^{14,32,33}$.

In China, some studies found that there were no significant differences in hospital mortality ${ }^{34-36}$, length of hospital stay ${ }^{37}$, and hospital costs ${ }^{34,37}$ among inpatients with MRSA and MSSA colonization or infection. Only one study explored the differences in the hospital mortality rate and length of hospital stay between MRSA cases and those without an S. aureus infection in China ${ }^{38}$. Therefore, the clinical and economic impact of MRSA remains largely uninvestigated in China. In this study, we aimed to compare the clinical and economic differences between MRSA and MSSA cases and between MRSA cases and those without an S. aureus infection. We also performed the analyses on both surviving and non-surviving patients.

\section{Materials and Methods}

Study site. This study was conducted in four tertiary hospitals in China: one in Shandong Province (site 3) and three in Zhejiang Province (site 1, site 2 and site 4). Site 1 is a combined traditional Chinese and Western medicine provincial hospital, site 2 is a general county hospital, and sites 3 and 4 are general provincial hospitals. The numbers of beds were $1,800,1,727,3,500$ and 3,200, respectively, and the numbers of discharged patients were approximately 49,000,76,600,140,000 and 154,000, respectively. We chose to include these hospitals because of their relatively complete hospital information systems, which can make reliable data collection possible.

Patients and data collection. This was a retrospective and multicentre study. First, we included $100 \%$ of inpatients in hospital sites 1-3 and 60\% of inpatients in hospital site 4 between 2013 and 2015 who had clinical samples positive for MRSA colonization or infection. The control group comprised clinical samples from inpatients with MSSA colonization or infection-positive clinical samples and inpatients without an S. aureus infection who were hospitalized during the same study period. To avoid duplication, only patients colonized and infected with the first episode of $S$. aureus detected were included in this study. Methicillin resistance was defined as resistance to anti-staphylococcal $\beta$-lactam antibiotics or cephamycins (oxacillin or cefoxitin) ${ }^{2}$.

Inpatient characteristics were collected from electronic medical records. Variables and dates relating to demographics (age, sex, and insurance), comorbidities (disease diagnosis, and Charlson comorbidity index $\left.(\mathrm{CCI})^{39}\right)$, hospital events (admitting service, surgical services, and dates of hospital and intensive care unit (ICU) admission/discharge), microbiological data, and clinical outcomes (death or alive during hospitalization) were extracted. Hospital costs were collected from electronic medical records as well.

Propensity score matching. We conducted propensity score matching (PSM) using STATA to eliminate selection bias by balancing the potential confounding variables between MRSA and MSSA cases and between MRSA cases and those without an S. aureus infection ${ }^{40,41}$. We employed 1:1 nearest-neighbour matching to obtain matched pairs. MRSA was the dependent variable, and inpatient characteristics (age, sex and insurance), comorbidities, CCI, number of diagnoses, admission to the ICU, and surgery were independent variables. Then, we analysed the hospital cost, length of hospital stay, and hospital mortality rate using generated pairs.

Indicators and data analyses. In this study, the main indicators included hospital costs, length of hospital stay, and hospital mortality rate. The total hospital cost for each inpatient's hospitalization was calculated, including the costs paid by both inpatients and health insurance, that is, the costs of health care. It comprised medication cost (antibiotic cost), diagnostic cost, treatment cost, material cost, and other costs. All hospital costs were presented in 2015 United States (US) \$ values using the 2015 consumer price index of China and the purchasing power parities $^{42,43}$.

Hospital costs, length of hospital stay, and hospital mortality rate between MRSA and MSSA cases and between MRSA cases and those without an S. aureus infection were compared using Wilcoxon rank-sum test and $\chi^{2}$ test and were described using median (95\% confidence interval (CI)) and proportion (95\% CI) for quantitative and qualitative variables, respectively. We also calculated the differences in hospital costs, length of hospital stay, and hospital mortality rate between the two groups, which are the incremental values due to MRSA. All tests were two-tailed, and p-values $<0.05$ were determined to be statistically significant. Data analyses were performed with SAS software.

Ethical approval and informed consent. The study was approved by the institutional review board of Zhejiang University School of Public Health, who waived the need for informed consent. All inpatients data were anonymized prior to analysis.

\section{Results}

A total of 1,335 inpatients with MRSA, 1,397 with MSSA, and 33,606 without an S. aureus infection were included in our study. Most of the inpatients were male (approximately 60\%) and were covered by insurance (approximately $80 \%$ ), and a small number of inpatients were admitted to the ICU or underwent surgery. The median ages were 74,66 , and 67 years old, the median number of diagnoses was 7, 6, and 5, and the median CCIs were 5, 4, and 4 for MRSA cases, MSSA cases, and those without an S. aureus infection, respectively (Tables 1-2).

Compared with both inpatients with MSSA and those without an S. aureus infection, those with MRSA were significantly associated with an older age, a higher proportion of males, a higher number of diagnoses, a higher CCI, and a higher rate of admission to the ICU (Tables 1-2). In addition, MRSA patients were more likely to have insurance coverage and undergo surgery than those without an S. aureus infection (Table 2). Some comorbidities between inpatients with MRSA and MSSA and between MRSA patients and those without an S. aureus infection 


\begin{tabular}{|c|c|c|c|c|c|c|}
\hline \multirow[b]{2}{*}{ Characteristics } & \multicolumn{3}{|l|}{ Before PSM } & \multicolumn{3}{|l|}{ After PSM } \\
\hline & MSSA & MRSA & P-value & MSSA & MRSA & P-value \\
\hline Number of inpatients, $\mathrm{n}$ & 1397 & 1335 & & 954 & 954 & \\
\hline Age in years, median (range) & $66(0-99)$ & $74(0-102)$ & $<0.000$ & $71(1-99)$ & $72(0-98)$ & 0.979 \\
\hline Sex male, $\mathrm{n}(\%)$ & $864(61.85)$ & $942(70.56)$ & $<0.000$ & $645(67.61)$ & $637(66.77)$ & 0.696 \\
\hline Insurance, $\mathrm{n}(\%)$ & $1133(81.10)$ & $1049(78.58)$ & 0.100 & $769(80.61)$ & $771(80.82)$ & 0.908 \\
\hline Number of diagnosis, median (range) & $6(1-24)$ & $7(1-37)$ & $<0.000$ & $6(1-24)$ & $6(1-22)$ & 0.670 \\
\hline Charlson comorbidity index, median (range) & $4(1-39)$ & $5(1-27)$ & 0.000 & $5(1-34)$ & $5(1-27)$ & 0.694 \\
\hline Admission to ICU, n(\%) & $148(10.59)$ & $352(26.37)$ & $<0.000$ & $143(14.99)$ & $123(12.89)$ & 0.186 \\
\hline Surgery, n(\%) & $443(31.71)$ & $410(30.71)$ & 0.573 & $293(30.71)$ & $280(29.35)$ & 0.516 \\
\hline Myocardial infarction, $\mathrm{n}(\%)$ & $29(2.08)$ & $27(2.02)$ & 0.922 & $17(1.78)$ & $19(1.99)$ & 0.736 \\
\hline Congestive heart failure, $\mathrm{n}(\%)$ & $204(14.60)$ & $231(17.30)$ & 0.054 & $154(16.14)$ & $149(15.62)$ & 0.754 \\
\hline Peripheral vascular disease, $\mathrm{n}(\%)$ & $15(1.07)$ & $16(1.20)$ & 0.758 & $13(1.36)$ & $9(0.94)$ & 0.391 \\
\hline Cerebrovascular diseases, $\mathrm{n}(\%)$ & $652(46.67)$ & $720(53.93)$ & $<0.000$ & $506(53.04)$ & $511(53.56)$ & 0.819 \\
\hline Dementia, $\mathrm{n}(\%)$ & $55(3.94)$ & $70(5.24)$ & 0.102 & $42(4.40)$ & $60(6.29)$ & 0.067 \\
\hline Chronic pulmonary disease, n(\%) & $210(15.03)$ & $289(21.65)$ & $<0.000$ & $179(18.76)$ & $172(18.03)$ & 0.679 \\
\hline Connective tissue disease, $\mathrm{n}(\%)$ & $43(3.08)$ & $18(1.35)$ & 0.002 & $17(1.78)$ & $16(1.68)$ & 0.861 \\
\hline Mild liver disease, $\mathrm{n}(\%)$ & $59(4.22)$ & $41(3.07)$ & 0.109 & $35(3.67)$ & $35(3.67)$ & 1.000 \\
\hline Peptic ulcer disease, $\mathrm{n}(\%)$ & $28(2.00)$ & $53(3.97)$ & 0.002 & $26(2.73)$ & $24(2.52)$ & 0.774 \\
\hline Diabetes mellitus, $\mathrm{n}(\%)$ & $369(26.41)$ & $364(27.27)$ & 0.615 & $250(26.21)$ & $258(27.04)$ & 0.679 \\
\hline Diabetes mellitus with chronic complications, n(\%) & $49(3.51)$ & $26(1.95)$ & 0.013 & $23(2.41)$ & $22(2.31)$ & 0.880 \\
\hline Moderate to severe chronic kidney disease, $\mathrm{n}(\%)$ & $102(7.30)$ & $130(9.74)$ & 0.022 & $84(8.81)$ & $84(8.81)$ & 1.000 \\
\hline Hemiplegia, $\mathrm{n}(\%)$ & $14(1.00)$ & $27(2.02)$ & 0.028 & $10(1.05)$ & $6(0.63)$ & 0.315 \\
\hline Solid tumor without metastases, n(\%) & $130(9.31)$ & $97(7.27)$ & 0.054 & $74(7.76)$ & $76(7.97)$ & 0.865 \\
\hline Leukemia, $\mathrm{n}(\%)$ & $27(1.93)$ & $24(1.80)$ & 0.794 & $20(2.10)$ & $19(1.99)$ & 0.871 \\
\hline Malignant lymphoma, n(\%) & $18(1.29)$ & $17(1.27)$ & 0.972 & $15(1.57)$ & $15(1.57)$ & 1.000 \\
\hline Severe liver disease, $\mathrm{n}(\%)$ & $20(1.43)$ & $18(1.35)$ & 0.853 & $17(1.78)$ & $14(1.47)$ & 0.587 \\
\hline Metastatic tumor, n(\%) & $106(7.59)$ & $39(2.92)$ & $<0.000$ & $39(4.09)$ & $39(4.09)$ & 1.000 \\
\hline
\end{tabular}

Table 1. Characteristics of the patients with MRSA and MSSA before and after PSM.

were significantly different. Therefore, PSM was conducted to balance the characteristics between the two groups, and 954 and 1,313 pairs, respectively, were obtained (Tables 1-2). These pairs were subjected to analysis of the hospital costs, length of hospital stay, and hospital mortality rate. After PSM, there were no differences in baseline characteristics between the two groups.

Inpatients with MRSA were significantly associated with higher hospital costs than those with MSSA. The median differences (95\% CI) in total hospital cost, antibiotic cost, medication cost, diagnostic cost, treatment cost, material cost, and other costs were $\$ 3,220$ (\$3,103-\$3,393), \$672 (\$579-\$723), \$2,368 (\$2,157-\$2,633), \$255 (\$253-\$278), \$403 (\$369-\$413), \$268 (\$278-\$295), and \$8 (\$6-\$9), respectively. For surviving inpatients, the median differences in hospital costs were $\$ 3,182, \$ 653, \$ 2,373, \$ 243, \$ 429, \$ 271$, and $\$ 7$, respectively. For non-surviving inpatients, there were significant differences in antibiotic cost, medication cost, and diagnostic cost, with median differences of $\$ 2,318, \$ 3,078$, and $\$ 382$, respectively (Table 3 ).

Inpatients with MRSA were significantly associated with higher hospital costs than those without an $S$. aureus infection. The median differences (95\% CI) in total hospital cost, antibiotic cost, medication cost, diagnostic cost, treatment cost, material cost, and other costs were $\$ 9,606(\$ 9,162-\$ 10,575), \$ 1,112(\$ 1,021-\$ 1,210), \$ 5,516$ (\$5,148-\$5,870), \$877 (\$797-\$986), \$1,835 (\$1,677-\$1,975), \$671 (\$621-\$725), and \$7 (\$6-\$9), respectively. For surviving inpatients, the median differences in hospital costs were $\$ 9,336, \$ 31,065, \$ 5,354, \$ 817, \$ 1,771, \$ 654$, and $\$ 6$, respectively. For non-surviving inpatients, there were significant differences in hospital costs except other costs, with median differences of $\$ 13,855, \$ 3,631, \$ 8,047, \$ 1,851, \$ 2,588$, and $\$ 826$, respectively (Table 4).

The length of hospital stay was significantly longer for inpatients with MRSA than for inpatients with MSSA (27 days vs 21 days). There was a significant difference among surviving inpatients, with a median difference of 6 days, but no difference in non-surviving inpatients between the two groups $(\mathrm{P}=0.0972)$. Compared with inpatients without an S. aureus infection, whether being a survivor or non-survivor, those with MRSA were significantly associated with a longer length of hospital stay, with median differences of 14 and 19 days, respectively (Table 5).

Notably, regarding the hospital mortality rate, the difference between MRSA and MSSA inpatients was not significant $(\mathrm{P}=0.265)$; however, there was a significant difference between MRSA cases and those without an $S$. aureus infection $(4.80 \%$ (3.64-5.96\%) vs $1.22 \%$ (0.63-1.81\%), respectively) (Table 6).

\section{Discussion}

To the best of our knowledge, this is the first multicentre study with a large sample size exploring the clinical and economic impact of MRSA in mainland China using the PSM method. It is also the first study to quantify the clinical and economic outcome of MRSA by comparing both MSSA inpatients and those without an $S$. aureus infection. It might be that the effect of methicillin resistance on clinical and economic outcomes among 


\begin{tabular}{|c|c|c|c|c|c|c|}
\hline \multirow[b]{2}{*}{ Characteristics } & \multicolumn{3}{|l|}{ Before PSM } & \multicolumn{3}{|l|}{ After PSM } \\
\hline & $\begin{array}{l}\text { Without an } \\
\text { S. aureus }\end{array}$ & MRSA & P-value & $\begin{array}{l}\text { Without an } \\
\text { S. aureus }\end{array}$ & MRSA & P-value \\
\hline Number of inpatients, $\mathrm{n}$ & 33606 & 1335 & & 1313 & 1313 & \\
\hline Age in years, median (range) & $67(0-102)$ & $74(0-102)$ & $<0.000$ & $72(0-102)$ & $73(0-102)$ & 0.762 \\
\hline Sex male, $\mathrm{n}(\%)$ & 20565 (61.19) & $942(70.56)$ & $<0.000$ & $917(69.84)$ & $924(70.37)$ & 0.765 \\
\hline Insurance, $\mathrm{n}(\%)$ & $27249(81.08)$ & $1049(78.58)$ & 0.022 & $1015(77.30)$ & $1033(78.67)$ & 0.397 \\
\hline Number of diagnosis, median (range) & $5(1-25)$ & $7(1-37)$ & $<0.000$ & $7(1-25)$ & $7(1-37)$ & 0.793 \\
\hline Charlson comorbidity index, median (range) & $4(1-37)$ & $5(1-27)$ & $<0.000$ & $5(1-25)$ & $5(1-27)$ & 0.846 \\
\hline Admission to ICU, $\mathrm{n}(\%)$ & $1284(3.82)$ & $352(26.37)$ & $<0.000$ & $337(25.67)$ & $333(25.36)$ & 0.858 \\
\hline Surgery, $\mathrm{n}(\%)$ & $7453(22.18)$ & $410(30.71)$ & $<0.000$ & $420(31.99)$ & $407(31.00)$ & 0.585 \\
\hline Myocardial infarction, n(\%) & $659(1.96)$ & $27(2.02)$ & 0.874 & $24(1.83)$ & $25(1.90)$ & 0.885 \\
\hline Congestive heart failure, $\mathrm{n}(\%)$ & $6451(19.20)$ & $231(17.30)$ & 0.085 & $201(15.31)$ & $228(17.36)$ & 0.154 \\
\hline Peripheral vascular disease, $\mathrm{n}(\%)$ & $278(0.83)$ & $16(1.20)$ & 0.145 & $23(1.75)$ & $16(1.22)$ & 0.259 \\
\hline Cerebrovascular diseases, $\mathrm{n}(\%)$ & $10863(32.32)$ & $720(53.93)$ & $<0.000$ & $709(54.00)$ & $701(53.39)$ & 0.754 \\
\hline Dementia, $\mathrm{n}(\%)$ & $225(0.67)$ & $70(5.24)$ & $<0.000$ & $65(4.95)$ & $67(5.10)$ & 0.858 \\
\hline Chronic pulmonary disease, $\mathrm{n}(\%)$ & $11876(35.34)$ & $289(21.65)$ & $<0.000$ & $290(22.09)$ & $288(21.93)$ & 0.925 \\
\hline Connective tissue disease, n(\%) & $1122(3.34)$ & $18(1.35)$ & $<0.000$ & $23(1.75)$ & $18(1.37)$ & 0.431 \\
\hline Mild liver disease, $\mathrm{n}(\%)$ & $1604(4.77)$ & $41(3.07)$ & 0.004 & $45(3.43)$ & $41(3.12)$ & 0.661 \\
\hline Peptic ulcer disease, $\mathrm{n}(\%)$ & $986(2.93)$ & $53(3.97)$ & 0.029 & $46(3.50)$ & $52(3.96)$ & 0.537 \\
\hline Diabetes mellitus, $\mathrm{n}(\%)$ & $7045(20.96)$ & $364(27.27)$ & $<0.000$ & $357(27.19)$ & $355(27.04)$ & 0.930 \\
\hline Diabetes mellitus with chronic complications, n(\%) & $890(2.65)$ & $26(1.95)$ & 0.116 & $27(2.06)$ & $26(1.98)$ & 0.890 \\
\hline Moderate to severe chronic kidney disease, $\mathrm{n}(\%)$ & $2507(7.46)$ & $130(9.74)$ & 0.002 & $127(9.67)$ & $126(9.60)$ & 0.947 \\
\hline Hemiplegia, n(\%) & $89(0.26)$ & $27(2.02)$ & $<0.000$ & $2491.83)$ & $25(1.90)$ & 0.885 \\
\hline Solid tumor without metastases, n(\%) & $3845(11.44)$ & $97(7.27)$ & $<0.000$ & $102(7.77)$ & $96(7.31)$ & 0.657 \\
\hline Leukemia, $\mathrm{n}(\%)$ & $760(2.26)$ & $24(1.80)$ & 0.262 & $27(2.06)$ & $24(1.83)$ & 0.671 \\
\hline Malignant lymphoma, n(\%) & $536(1.59)$ & $17(1.27)$ & 0.356 & $26(1.98)$ & $17(1.29)$ & 0.166 \\
\hline Severe liver disease, $\mathrm{n}(\%)$ & $300(0.89)$ & $18(1.35)$ & 0.086 & $19(1.45)$ & $18(1.37)$ & 0.868 \\
\hline Metastatic tumor, n(\%) & $1350(4.02)$ & $39(2.92)$ & $<0.000$ & $38(2.89)$ & $39(2.97)$ & 0.908 \\
\hline
\end{tabular}

Table 2. Characteristics of the patients with MRSA and those without an S. aureus infection before and after PSM.

inpatients with $S$. aureus is underestimated after adjusting for the confounding factors between the MRSA and MSSA groups. Compared with inpatients without an S. aureus infection, however, the effect of MRSA is balanced. We found that after PSM, MRSA is associated with an increased total hospital cost ranging from $\$ 3,220$ to $\$ 9,606$, an excess length of hospital stay of 6 days-14 days, and an attributable hospital mortality rate of 0-3.58\%.

Among non-surviving inpatients, methicillin resistance does not independently increase the total hospital cost or length of hospital stay when the control group is MSSA. However, when the control group was inpatients without an S. aureus infection, MRSA was an independent factor for the total hospital cost or length of hospital stay among both survivors and non-survivors. Some studies reported that the difference in the total hospital cost and length of hospital stay for MRSA inpatients, compared with MSSA inpatients, are not significant ${ }^{11,34,37}$; however, compared with inpatients without an $S$. aureus infection, they are significant ${ }^{6,21,32}$, which is consistent with our findings. Some studies conducted in China reported a 7.3 times higher median total hospital cost and 14 days longer median length of hospital stay among inpatients with MRSA than those without an S. aureus infection after controlling for some confounding factors ${ }^{11}$; some studies only found significant differences between MRSA and MSSA patients using a univariate analysis, but not using a multivariate analysis ${ }^{34,35}$.

The length of hospital stay is a major contributing factor to hospital costs ${ }^{23}$. In addition to an increase in daily bed cost, the prolonged length of hospital stay may be related to more treatment and diagnostic services, which yield substantial hospital costs; therefore, we did not include length of hospital stay in PSM analyses ${ }^{44}$. In the case of inpatients that die during hospitalization, their illness was more likely to be associated with critical illness; thus, there is a greater possibility that they will be admitted to the ICU, undergo more surgeries, and be treated with more diagnoses and more expensive medications, and these might contribute to increased hospital costs and length of hospital stay compared to surviving inpatients, but it this can attenuate the influence of methicillin resistance. Thus, we only found a significant difference in the total hospital cost and length of hospital stay among surviving inpatients between the MRSA and MSSA groups.

The hospital mortality rate between MRSA and MSSA inpatients has long been controversial, and the results in some studies are inconsistent ${ }^{12,16,17,20,33,40,45,46}$. It was reported that patients with MRSA pneumonia infections had a higher mortality rate than those with MSSA $(\mathrm{P}<0.001)^{13}$. In our study, we found that there were no differences in the hospital mortality rate between MRSA and MSSA inpatients; however, a significant difference existed between MRSA inpatients and those without an S. aureus infection, and this is consistent with some other studies conducted in China ${ }^{34-36,38}$. Some studies in China reported a significantly higher hospital mortality for 


\begin{tabular}{|c|c|c|c|c|c|c|c|c|c|c|}
\hline \multirow{2}{*}{$\begin{array}{l}\text { Hospital cost (\$, } \\
\text { 2015) }\end{array}$} & \multicolumn{3}{|l|}{ MSSA } & \multicolumn{3}{|l|}{ MRSA } & \multicolumn{3}{|c|}{ Difference } & \multirow[b]{2}{*}{ P-value } \\
\hline & Median & \multicolumn{2}{|l|}{ 95\% CI } & Median & \multicolumn{2}{|l|}{ 95\% CI } & Median & \multicolumn{2}{|l|}{ 95\% CI } & \\
\hline \multicolumn{11}{|c|}{ Survivor+non-survivor (\%) } \\
\hline Total hospital cost & 8,230 & 7,431 & 9,131 & 11,450 & 10,534 & 12,525 & 3,220 & 3,103 & 3,393 & $<0.000$ \\
\hline Antibiotic cost & 391 & 348 & 452 & 1,063 & 927 & 1,175 & 672 & 579 & 723 & $<0.000$ \\
\hline Medication cost & 3,460 & 3,087 & 3,774 & 5,828 & 5,244 & 6,407 & 2,368 & 2,157 & 2,633 & $<0.000$ \\
\hline Diagnostic cost & 1,321 & 1,242 & 1,400 & 1,577 & 1,495 & 1,678 & 255 & 253 & 278 & $<0.000$ \\
\hline Treatment cost & 1,832 & 1,656 & 2,053 & 2,235 & 2,025 & 2,466 & 403 & 369 & 413 & $<0.000$ \\
\hline Materical cost & 464 & 405 & 520 & 733 & 683 & 814 & 268 & 278 & 295 & $<0.000$ \\
\hline Other cost & 10 & 8 & 12 & 18 & 15 & 21 & 8 & 6 & 9 & 0.0001 \\
\hline \multicolumn{11}{|l|}{ Survivor (\%) } \\
\hline Total hospital cost & 7,995 & 7,314 & 8,833 & 11,178 & 10,357 & 12,155 & 3,182 & 3,042 & 3,322 & $<0.000$ \\
\hline Antibiotic cost & 384 & 335 & 438 & 1,037 & 911 & 1,156 & 653 & 577 & 717 & $<0.000$ \\
\hline Medication cost & 3,305 & 2,961 & 3,657 & 5,678 & 5,068 & 6,262 & 2,373 & 2,107 & 2,604 & $<0.000$ \\
\hline Diagnostic cost & 1,311 & 1,222 & 1,383 & 1,554 & 1,462 & 1,656 & 243 & 239 & 273 & $<0.000$ \\
\hline Treatment cost & 1,794 & 1,627 & 2,045 & 2,223 & 2,020 & 2,467 & 429 & 393 & 422 & $<0.000$ \\
\hline Materical cost & 452 & 398 & 512 & 723 & 668 & 788 & 271 & 270 & 276 & $<0.000$ \\
\hline Other cost & 9 & 8 & 12 & 17 & 14 & 20 & 7 & 6 & 9 & 0.0001 \\
\hline \multicolumn{11}{|l|}{ Non-survivor (\%) } \\
\hline Total hospital cost & 11,535 & 7,784 & 17,975 & 18,069 & 12,965 & 21,375 & 6,533 & 5,181 & 3,400 & 0.0594 \\
\hline Antibiotic cost & 824 & 506 & 1,530 & 3,141 & 1,032 & 6,203 & 2,318 & 527 & 4,673 & 0.0026 \\
\hline Medication cost & 6,754 & 5,217 & 8,135 & 9,832 & 7,113 & 13,268 & 3,078 & 1,896 & 5,133 & 0.0206 \\
\hline Dignostic cost & 2,227 & 1,135 & 2,740 & 2,610 & 1,786 & 3,749 & 382 & 651 & 1,009 & 0.0498 \\
\hline Treatment cost & 2,459 & 1,091 & 4,127 & 2,438 & 1,638 & 4,622 & -21 & 548 & 496 & 0.2601 \\
\hline Materical cost & 633 & 457 & 1,645 & 928 & 751 & 1,527 & 295 & 295 & -118 & 0.2057 \\
\hline Other cost & 31 & 18 & 51 & 30 & 24 & 75 & 0 & 6 & 23 & 0.6523 \\
\hline
\end{tabular}

Table 3. Hospital costs of patients with MRSA and MSSA after PSM for potential confounding variables.

\begin{tabular}{|c|c|c|c|c|c|c|c|c|c|c|}
\hline \multirow{2}{*}{$\begin{array}{l}\text { Hospital cost (\$, } \\
2015)\end{array}$} & \multicolumn{3}{|c|}{ Without an S. aureus } & \multicolumn{3}{|l|}{ MRSA } & \multicolumn{3}{|c|}{ Difference } & \multirow[b]{2}{*}{ P-value } \\
\hline & Median & \multicolumn{2}{|c|}{$95 \% \mathrm{CI}$} & Median & \multicolumn{2}{|l|}{$95 \% \mathrm{CI}$} & Median & \multicolumn{2}{|l|}{$95 \% \mathrm{CI}$} & \\
\hline \multicolumn{11}{|c|}{ Survivor+non-survivor (\%) } \\
\hline Total hospital cost & 4,241 & 3,834 & 4,818 & 13,847 & 12,996 & 15,393 & 9,606 & 9,162 & 10,575 & $<0.000$ \\
\hline Antibiotic cost & 225 & 197 & 255 & 1,336 & 1,218 & 1,465 & 1,112 & 1,021 & 1,210 & $<0.000$ \\
\hline Medication cost & 1,619 & 1,443 & 1,805 & 7,136 & 6,591 & 7,675 & 5,516 & 5,148 & 5,870 & $<0.000$ \\
\hline Diagnostic cost & 988 & 950 & 1,030 & 1,866 & 1,746 & 2,017 & 877 & 797 & 986 & $<0.000$ \\
\hline Treatment cost & 896 & 816 & 1,005 & 2,731 & 2,493 & 2,980 & 1,835 & 1,677 & 1,975 & $<0.000$ \\
\hline Materical cost & 237 & 199 & 290 & 908 & 820 & 1,016 & 671 & 621 & 725 & $<0.000$ \\
\hline Other cost & 14 & 12 & 16 & 21 & 18 & 24 & 7 & 6 & 9 & 0.0125 \\
\hline \multicolumn{11}{|l|}{ Survivor (\%) } \\
\hline Total hospital cost & 4,179 & 3,809 & 4,785 & 13,516 & 12,655 & 14,566 & 9,336 & 8,846 & 9,782 & $<0.000$ \\
\hline Antibiotic cost & 228 & 199 & 255 & 1,293 & 1,175 & 1,408 & 1,065 & 977 & 1,153 & $<0.000$ \\
\hline Medication cost & 1,606 & 1,422 & 1,770 & 6,960 & 6,430 & 7,485 & 5,354 & 5,008 & 5,715 & $<0.000$ \\
\hline Diagnostic cost & 988 & 945 & 1,030 & 1,804 & 1,694 & 1,967 & 817 & 748 & 937 & $<0.000$ \\
\hline Treatment cost & 893 & 810 & 994 & 2,664 & 2,445 & 2,941 & 1,771 & 1,634 & 1,947 & $<0.000$ \\
\hline Materical cost & 234 & 191 & 282 & 888 & 785 & 997 & 654 & 593 & 716 & $<0.000$ \\
\hline Other cost & 14 & 12 & 15 & 20 & 17 & 23 & 6 & 5 & 8 & 0.0413 \\
\hline \multicolumn{11}{|l|}{ Non-survivor (\%) } \\
\hline Total hospital cost & 5,920 & 4,592 & 12,683 & 19,775 & 16,289 & 24,190 & 13,855 & 11,698 & 11,507 & 0.0009 \\
\hline Antibiotic cost & 170 & 74 & 432 & 3,802 & 2,423 & 5,980 & 3,631 & 2,349 & 5,548 & $<0.000$ \\
\hline Medication cost & 2,945 & 1,954 & 9,331 & 10,992 & 8,945 & 13,905 & 8,047 & 6,991 & 4,574 & 0.0011 \\
\hline Dignostic cost & 1,206 & 560 & 1,631 & 3,057 & 2,287 & 3,781 & 1,851 & 1,727 & 2,150 & 0.0003 \\
\hline Treatment cost & 1,457 & 507 & 2,182 & 4,045 & 2,577 & 5,513 & 2,588 & 2,070 & 3,331 & 0.0028 \\
\hline Materical cost & 507 & 230 & 1,589 & 1,333 & 930 & 1,930 & 826 & 700 & 341 & 0.0416 \\
\hline Other cost & 32 & 7 & 56 & 28 & 24 & 38 & -4 & 17 & -18 & 0.587 \\
\hline
\end{tabular}

Table 4. Hospital costs of patients with MRSA and those without an S. aureus infection after PSM for potential confounding variables. 


\begin{tabular}{|c|c|c|c|c|c|c|c|c|c|}
\hline \multirow{4}{*}{$\begin{array}{l}\text { Inpatients } \\
\text { MSSA }\end{array}$} & \multicolumn{9}{|c|}{ Length of hospital stay (days) } \\
\hline & \multicolumn{3}{|c|}{ Survivor+non-survivor } & \multicolumn{3}{|l|}{ Survivor } & \multicolumn{3}{|c|}{ Non-survivor } \\
\hline & \multirow{2}{*}{\begin{tabular}{|l} 
Median \\
21
\end{tabular}} & \multicolumn{2}{|c|}{$95 \% \mathrm{CI}$} & \multirow{2}{*}{\begin{tabular}{|l|} 
Median \\
21
\end{tabular}} & \multicolumn{2}{|c|}{ 95\% CI } & \multirow{2}{*}{\begin{tabular}{|l|} 
Median \\
20
\end{tabular}} & \multicolumn{2}{|c|}{ 95\% CI } \\
\hline & & 20 & 23 & & 20 & 23 & & 12 & 26 \\
\hline MRSA & 27 & 25 & 29 & 27 & 26 & 29 & 22 & 19 & 36 \\
\hline Difference & 6 & 5 & 6 & 6 & 6 & 6 & 2 & 7 & 10 \\
\hline P-value & \multicolumn{3}{|l|}{$<0.000$} & \multicolumn{3}{|l|}{$<0.000$} & \multicolumn{3}{|l|}{0.0972} \\
\hline $\begin{array}{l}\text { Without an } S \text {. } \\
\text { aureus }\end{array}$ & 14 & 13 & 14 & 14 & 13 & 14 & 8.5 & 4 & 29 \\
\hline MRSA & 28 & 26 & 29 & 28 & 26 & 29 & 27 & 21 & 34 \\
\hline Difference & 14 & 13 & 15 & 14 & 13 & 15 & 19 & 17 & 5 \\
\hline P-value & \multicolumn{3}{|l|}{$<0.000$} & \multicolumn{3}{|l|}{$<0.000$} & \multicolumn{3}{|l|}{0.0006} \\
\hline
\end{tabular}

Table 5. Length of hospital stay among inpatients with MRSA and MSSA and among inpatients with MRSA and those without an S. aureus infection after PSM for potential confounding variables.

\begin{tabular}{|c|c|c|c|c|}
\hline \multirow{3}{*}{$\begin{array}{l}\text { Inpatients } \\
\text { MSSA }\end{array}$} & \multicolumn{4}{|c|}{ Hospital mortality(\%) } \\
\hline & \multirow{2}{*}{\begin{tabular}{|c|} 
Median \\
3.98 \\
\end{tabular}} & \multicolumn{2}{|c|}{$95 \% \mathrm{CI}$} & P-value \\
\hline & & 2.74 & 5.22 & \multirow{3}{*}{0.265} \\
\hline MRSA & 3.04 & 1.95 & 4.13 & \\
\hline Difference & -0.94 & -0.79 & -1.09 & \\
\hline $\begin{array}{l}\text { Without an } S \text {. } \\
\text { aureus }\end{array}$ & 1.22 & 0.63 & 1.81 & \multirow{3}{*}{$<\mathbf{0 . 0 0 0}$} \\
\hline MRSA & 4.80 & 3.64 & 5.96 & \\
\hline Difference & 3.58 & 3.01 & 4.15 & \\
\hline
\end{tabular}

Table 6. Hospital mortality rate among inpatients with MRSA and MSSA and among inpatients with MRSA and those without an S. aureus infection after PSM for potential confounding variables.

MRSA patients than those without an S. aureus infection ( $10.94 \%$ vs $4.43 \%)^{38}$; however, there were no significant differences in hospital mortality between MRSA and MSSA patients after adjusting for confounding factors ${ }^{34-36}$.

A total of 67 non-surviving inpatients were included, including 29 inpatients with MRSA and 38 with MSSA. The small sample size may lack statistical power to detect significant differences; therefore, cohort studies with larger sample sizes among non-surviving inpatients in both the MRSA and MSSA groups are needed in future studies. In addition, MRSA and MSSA patients are usually empirically treated with vancomycin before the cultures are returned; however, when treated with vancomycin rather than beta-lactam agents, they showed a worse outcome for MSSA patients but a better outcome for MRSA patients ${ }^{47}$. Controlling the confounding factors using PSM can also be expected to bias the study towards a similar result between the MRSA and MSSA groups. Therefore, it is important to select variables in the PSM analysis. Some variables representing the severity of illness, including whether admitted to the ICU, whether undergoing surgery, complications or underlying disease, were included in the PSM analyses to decrease the influence of these variables on the main indicators; however, we excluded some variables that were directly related to hospital costs, such as length of hospital stay, length of ICU stay, and number of surgeries $^{44}$, which is consistent with some studies ${ }^{13,48}$. Some caution should be taken in the interpretation of these results.

The average total hospital cost, length of hospital stay, and hospital mortality were $\$ 3,042$ and $\$ 2,470,10.1$ days and 9.4 days, $0.3 \%$ and $0.4 \%$ in Zhejiang and Shandong Provinces in 2015, respectively, which were similar to the national level $(\$ 2,378,9.6$ days, and $0.4 \%)$. Therefore, we assumed that the attributable total hospital cost, length of hospital stay, and hospital mortality due to MRSA from four hospitals in China were approximate results representing the national level. We also needed to expand this study to different types of hospitals in different areas in the future.

Certain limitations are worth considering in this study. First, due to the retrospective nature of our study, it is difficult to distinguish between colonization and infection, and ascertainment bias cannot be neglected. A stronger analysis needs to be conducted only in inpatients with a clinical infection in the future. In addition, in the PSM method, we only accounted for observable covariates regardless of other unobservable covariates; thus, there may remain some hidden biases after matching. Moreover, although it is a multicentre study, the data are from tertiary hospitals only and may not apply to other types of medical institutions.

\section{Conclusions}

Between the MRSA and MSSA groups, MRSA colonization or infection was significantly associated with a higher total hospital cost and longer length of hospital stay among survivors but not among non-survivors. There were, however, no differences in the hospital mortality rate. Between the MRSA group and the $S$. aureus-free group, MRSA colonization or infection was significantly associated with an increased total hospital cost, a prolonged length of hospital stay and a higher hospital mortality rate among both survivors and non-survivors. It is critical to quantify the clinical and economic impact of MRSA to justify resource allocation for the development of strategies to improve clinical outcomes and to reduce the economic burden. 
Received: 25 July 2019; Accepted: 17 February 2020;

Published online: 03 March 2020

\section{References}

1. Centers for Disease Control and Prevention, Antibiotic resistance threats in the United States, 2013. https://www.cdc.gov/ drugresistance/pdf/ar-threats-2013-508.pdf. (February 6) (2013).

2. Magiorakos, A. P. et al. Multidrug-resistant, extensively drug-resistant and pandrug-resistant bacteria: an international expert proposal for interim standard definitions for acquired resistance. Clinical Microbiology and Infection. 18, 268-281 (2012).

3. World Health Organization, Antimicrobial resistance global report on surveillance. https://www.who.int/drugresistance/ documents/surveillancereport/en/. (December 11) (2014).

4. Hu, F. et al. Resistance trends among clinical isolates in China reported from CHINET surveillance of bacterial resistance, 20052014. Clinical Microbiology and Infection. 221, S9-S14 (2016).

5. China Antimicrobial Resistance Surveillance System, Annual report of the China antimicrobial resistance surveillance, 2017. http:// www.carss.cn/Report/Details?aId=552. (February 14) (2018).

6. Anderson, D. J. et al. Clinical and financial outcomes due to methicillin resistant Staphylococcus aureus surgical site infection: a multi-center matched outcomes study. Plos One. 4, e8305 (2009).

7. Capitano, B., Leshem, O. A., Nightingale, C. H. \& Nicolau, D. P. Cost effect of managing methicillin-resistant Staphylococcus aureus in a long-term care facility. Journal of the American Geriatrics Society. 51, 10-16 (2003).

8. de Kraker, M. E. A., Davey, P. G. \& Grundmann, H. Mortality and hospital stay associated with resistant Staphylococcus aureus and Escherichia coli bacteremia: estimating the burden of antibiotic resistance in Europe. Plos Medicine. 8, (2011).

9. Engemann, J. J. et al. Adverse clinical and economic outcomes attributable to methicillin resistance among patients with Staphylococcus aureus surgical site infection. Clinical Infectious Diseases. 36, 592-598 (2003).

10. Filice, G. A. et al. Excess costs and utilization associated with methicillin resistance for patients with Staphylococcus aureus infection. Infection Control and Hospital Epidemiology. 31, 365-373 (2010).

11. Fu, J. et al. Case-control study of influence of methicillin-resistant Staphylococcus aureus infection on economic losses and length of hospital stay. Chinese Journal of Nosocomiology. 24, 2363-2365 (2014).

12. Guo, Y. et al. Study of attributive hospitalized cost and length of stay for hospital acquired infection due to multidrug resistance and none-resistance Acinetobacter baumannii. Chinese Journal of Health Statistics. 34, 378-381 (2017).

13. Klein, E. Y. et al. National costs associated with methicillin-susceptible and methicillin-resistant Staphylococcus aureus hospitalizations in the United States, 2010-2014. Clinical Infectious Diseases. 68, 22-28 (2019).

14. Lee, Y. J. et al. Impact of active screening for methicillin-resistant Staphylococcus aureus (MRSA) and decolonization on MRSA infections, mortality and medical cost: a quasi-experimental study in surgical intensive care unit. Critical Care. 19, 143 (2015).

15. Lodise, T. P. \& McKinnon, P. S. Clinical and economic impact of methicillin resistance in patients with Staphylococcus aureus bacteremia. Diagnostic Microbiology and Infectious Disease. 52, 113-122 (2005).

16. McHugh, C. G. \& Riley, L. W. Risk factors and costs associated with methicillin-resistant Staphylococcus aureus bloodstream infections. Infection Control and Hospital Epidemiology. 25, 425-430 (2004).

17. Nelson, R. E. et al. The impact of healthcare-associated methicillin-resistant Staphylococcus aureus infections on post-discharge healthcare costs and utilization. Infection Control and Hospital Epidemiology. 36, 534-542 (2015).

18. Ott, E. et al. Costs of nosocomial pneumonia caused by meticillin-resistant Staphylococcus aureus. Journal of Hospital Infection. 76, 300-303 (2010).

19. Reed, S. D. et al. Costs and outcomes among hemodialysis-dependent patients with methicillin-resistant or methicillin-susceptible Staphylococcus aureus bacteremia. Infection Control and Hospital Epidemiology. 26, 175-183 (2005).

20. Resch, A., Wilke, M. \& Fink, C. The cost of resistance: incremental cost of methicillin-resistant Staphylococcus aureus (MRSA) in German hospitals. European Journal of Health Economics. 10, 287-297 (2009).

21. Shorr, A. F. et al. Clinical and economic outcomes for patients with health care-associated Staphylococcus aureus pneumonia. Journal of Clinical Microbiology. 48, 3258-3262 (2010).

22. Shorr, A. F. et al. Morbidity and cost burden of methicillin-resistant Staphylococcus aureus in early onset ventilator-associated pneumonia. Critical Care. 10, R97 (2006).

23. Thampi, N. et al. Multicenter study of health care cost of patients admitted to hospital with Staphylococcus aureus bacteremia: Impact of length of stay and intensity of care. American Journal of Infection Control. 43, 739-744 (2015).

24. Xu, B., Yuan, H. \& Yang, P. Evaluation of economic burden induced by multidrug-resistant bacteria related infections in a tertiary general hospital. Chinese Journal of Experimental and Clinical Infectious Diseases. 11, 455-459 (2017).

25. Zhen, X., Lundborg, C. S., Sun, X., Hu, X. \& Dong, H. Economic burden of antibiotic resistance in ESKAPE organisms: a systematic review. Antimicrobial Resistance and Infection Control. 8, 137 (2019).

26. Zhen, X., Lundborg, C. S., Sun, X., Hu, X. \& Dong, H. The clinical and economic impact of antibiotic resistance in China: a systematic review and meta-analysis. Antibiotics (Basel). 8 (2019).

27. Rubio-Terres, C., Garau, J., Grau, S. \& Martinez-Martinez, L. Cost of bacteraemia caused by methicillin-resistant vs. methicillinsusceptible Staphylococcus aureus in Spain: a retrospective cohort study. Clinical Microbiology and Infection. 16, 722-728 (2010).

28. Nelson, R. E. et al. Reducing time-dependent bias in estimates of the attributable cost of health care-associated methicillin-resistant Staphylococcus aureus infections: a comparison of three estimation strategies. Medical Care. 53, 827-834 (2015).

29. Itani, K. M. F. et al. Outcomes and management costs in patients hospitalized for skin and skin-structure infections. American Journal of Infection Control. 39, 42-49 (2011).

30. Park, S. Y., Son, J. S., Oh, I. H., Choi, J. M. \& Lee, M. S. Clinical impact of methicillin-resistant Staphylococcus aureus bacteremia based on propensity scores. Infection. 39, 141-147 (2011).

31. Branch-Elliman, W. et al. Health and economic burden of post-partum Staphylococcus aureus breast abscess. Plos One. 8, e73155 (2013).

32. de Kraker, M. E. et al. Clinical impact of antimicrobial resistance in European hospitals: excess mortality and length of hospital stay related to methicillin-resistant Staphylococcus aureus bloodstream infections. Antimicrobial Agents and Chemotherapy. 55, 1598-1605 (2011).

33. Kim, C. J. et al. The burden of nosocomial staphylococcus aureus bloodstream infection in South Korea: a prospective hospital-based nationwide study. BMC Infectious Diseases. 14, 590 (2014).

34. Chen, W. et al. Comparison in prognosis of hospital-acquired pneumonia due to methicillin resistant and methicillin sensitive Staphylococcus aureus: analysis of propensity score matching. Chinese Journal of Infection Control. 15, 299-303 (2016).

35. Zhou, L., Qiao, F., Huang, W. \& Zong, Z. Impact of methicillin resistant Staphylococcus aureus bloodstream infection on the prognosis of patients. Chinese Journal of Infection and Control. 14, 27-30 (2015).

36. Zhu, C., Liu, C., Wu, B., Wu, Q. \& Huang, D. Analysis of antibiotic resistance in the Staphylococcus aureus strains isolated from bloodstream infections and associated patient outcome. Chinese Journal of Infection and Chemotherapy. 16, 1-4 (2016).

37. Li, X. et al. Epidemiology and outcomes of complicated skin and soft tissue infections among inpatients in southern China from 2008 to 2013. PLoS One. 11, (2016). 
38. Zhang, Z., Zhang, J., Xie, F., Lou, G. \& Li, X. Analysis of drug resistance of Staphyloccus aureus isolated from bloodstream and risk factors of methicillin resistant strains. Laboratory Medicine and Clinic. 10, 2380-2382 (2013).

39. Quan, H. et al. Coding algorithms for defining comorbidities in ICD-9-CM and ICD-10 administrative data. Medical Care. 43, $1130-1139$ (2005).

40. Li, H., Graham, D. J. \& Majumdar, A. The impacts of speed cameras on road accidents: an application of propensity score matching methods. Accident, Analysis and Prevention. 60, 148-157 (2013).

41. Khoo, A. L. et al. Evaluation of a risk-guided strategy for empirical carbapenem use in febrile neutropenia. International Journal of Antimicrobial Agents. 52, 350-357 (2018).

42. Organisation for Economic Cooperation and Development, Consumer price indices. https://stats.oecd.org/index. aspx?queryid $=221 \#$. (February 15) (2019).

43. Organisation for Economic Cooperation and Development, Purchasing Power Parities for GDP. https://stats.oecd.org/index. aspx?queryid=221\#. (February 15) (2019).

44. Hemmige, V. \& David, M. Z. Effects of including variables such as length of stay in a propensity score analysis with costs as outcome. Clinical Infectious Diseases. 69, 2039-2040 (2019).

45. Yu, Y. Risk factors of nosocomial MRSA infection and molecular characteristics of Staphylococcus aureus isolated from blood: Suzhou University (2016)

46. Cosgrove, S. E. et al. The impact of methicillin resistance in Staphylococcus aureus bacteremia on patient outcomes: mortality, length of stay, and hospital charges. Infection Control and Hospital Epidemiology. 26, 166-174 (2005).

47. McDanel, J. S. et al. Comparative effectiveness of beta-lactams versus vancomycin for treatment of methicillin-susceptible Staphylococcus aureus bloodstream infections among 122 hospitals. Clinical Infectious Diseases. 61, 361-367 (2015).

48. Huang, W. et al. In-hospital medical costs of infections caused by carbapenem-resistant Klebsiella pneumoniae. Clinical Infectious Diseases. 672, S225-S230 (2018).

\section{Acknowledgements}

It is acknowledged that we obtained valuable help from the four hospitals for data collection. This study was supported by China Scholarship Council.

\section{Author contributions}

X.Z. and H.D. participated in the conception and design of the study, collection, analysis and interpretation of data, drafted and revised the manuscript. C.S.L. participated in the interpretation of data, revised the manuscript. M.Z., Y.L., X.S., X.H., S.G., Y.G. and J.W. participated in the data collection and analysis, revised the manuscript. All authors read and approved the final manuscript.

\section{Competing interests}

The authors declare no competing interests.

\section{Additional information}

Correspondence and requests for materials should be addressed to H.D.

Reprints and permissions information is available at www.nature.com/reprints.

Publisher's note Springer Nature remains neutral with regard to jurisdictional claims in published maps and institutional affiliations.

(c) (i) Open Access This article is licensed under a Creative Commons Attribution 4.0 International License, which permits use, sharing, adaptation, distribution and reproduction in any medium or format, as long as you give appropriate credit to the original author(s) and the source, provide a link to the Creative Commons license, and indicate if changes were made. The images or other third party material in this article are included in the article's Creative Commons license, unless indicated otherwise in a credit line to the material. If material is not included in the article's Creative Commons license and your intended use is not permitted by statutory regulation or exceeds the permitted use, you will need to obtain permission directly from the copyright holder. To view a copy of this license, visit http://creativecommons.org/licenses/by/4.0/.

(C) The Author(s) 2020 